\title{
Magnetic trapping of neutrons
}

\author{
P. R. Huffman*†, C. R. Brome* , J.S. Butterworth ${ }^{* \ddagger}$, K. J. Coakley ${ }^{\S}$, \\ M.S. Dewey ${ }^{\dagger}$, S. N. Dzhosyuk*, R. Golub", G. L. Greeneף, K. Habicht" ${ }^{\|}$, \\ S. K. Lamoreaux ${ }^{\Uparrow}$, C. E. H. Mattoni*, D. N. McKinsey*, F. E. Wietfeldt ${ }^{* \dagger}$, \\ \& J. M. Doyle* \\ * Harvard University, 17 Oxford Street, Cambridge, Massachusetts 02138, USA. \\ $\dagger$ National Institute of Standards and Technology, 100 Bureau Drive, MS 8461, Gaithersburg, Maryland 20899, \\ USA. \\ $\S$ National Institute of Standards and Technology, 325 Broadway, MS 898.02, Boulder, Colorado 80303, USA. \\ || Hahn-Meitner-Institut, Glienicker Straße 100, D-14109 Berlin, Germany. \\ ฯ University of California, Los Alamos National Laboratory, P.O. Box 1663, Los Alamos, New Mexico 87545, \\ USA.
}

Accurate measurement of the lifetime of the neutron (which is unstable to beta decay) is important for understanding the weak nuclear forcet and the creation of matter during the Big Bangl. Previous measurements of the neutron lifetime have mainly been limited by certain systematic errors; however, these could in principle be avoided by performing measurements on neutrons stored in a magnetic trap目. Neutral and charged particle traps are widely used tool for studying both composite and elementary particles, because they allow long interaction times and isolation from perturbing environment $\$$. Here we report the magnetic trapping of neutrons. The trapping region is filled with superfluid ${ }^{4} \mathrm{He}$, which is used to load neutrons into the trap and as a scintillator to detect their decay. Neutrons have a lifetime in the trap of $750_{-200}^{+330}$ seconds, mainly limited by their beta decay rather than trap losses. Our experiment verifies theoretical predictions regarding the loading process and magnetic trapping of neutrons. Further refinement of this method should lead to improved precision in the neutron lifetime measurement.

A more precisely known value of the neutron lifetime will benefit both weak interaction and Big Bang Nucleosynthesis (BBN) theory. Combined with the measured neutron beta-decay

\footnotetext{
${ }^{\ddagger}$ Present address: Institut Laue-Langevin, B.P. 156-6 rue Jules Horowitz, 38042, Grenoble (Cedex 9), France.
} 
asymmetry, the neutron lifetime yields the $V_{u d}$ element of the Cabibbo-Kobayashi-Maskawa (CKM) quark mixing matrix. Non-unitarity of the CKM matrix would indicate physics beyond the Standard Modell. In addition, the theoretical uncertainty in the predicted ${ }^{4} \mathrm{He}$ abundance in the BBN model is dominated by the uncertainty in the neutron lifetime 3 .

Many experiments, using different methods, have measured the neutron beta-decay lifetime日 $\mathrm{E}$. The most precise measurements use ultracold neutrons (UCN, neutrons with energies $E / k_{b} \leqslant 1 \mathrm{mK}$, where $k_{b}$ is Boltzmann's constant) 10 confined in a 'bottle' made of materials with high UCN reflectivity. The largest uncertainties are due to UCN interactions with the material walls and fluctuations in the initial filling densitiest 6 . Extrapolation methods are necessary to extract the neutron beta-decay lifetime. In another experiment, neutrons with specific trajectories and in a band of velocities (typically between 10-14 $\mathrm{m} \mathrm{s}^{-1}$ ) were kept in a magnetic storage ring similar to those used in high-energy particle physics $\mathrm{\theta}$. Since storage rings only confine neutrons in two dimensions, coupling between the large longitudinal momentum and small radial momentum by way of betatron oscillations allows neutrons to escape. In contrast, neutrons in magnetic traps are not susceptible to wall losses or betatron oscillations.

Static magnetic traps are formed by creating a magnetic field minimum in free space. The confining potential depth $(D)$ of such a trap is determined by the magnetic moment of the trapped species $(\mu)$ and the difference $(\Delta B)$ between the magnitude of the field at the edge of the trap and at the minimum, $D=\mu \Delta B$. A particle in a low-field-seeking state (one with its magnetic moment anti-parallel with the local magnetic field vector) is pushed towards the trap minimum. Low-field-seeking particles with total energy less than $D$ are energetically forbidden from leaving the trapping region. For atoms and molecules with a magnetic moment of one Bohr magneton it is possible to produce trap depths of $\sim 1 \mathrm{~K}$. The trap depth for a neutron in the same trap would be only $\sim 1 \mathrm{mK}$, because of its much smaller magnetic moment. Despite this difficulty, magnetic trapping of the neutron was proposed as early as 1961 by Vladimirskiil1 1 . His proposed technique was later used to confine neutrons using a combination of gravity and magnet12. A separate effort to trap neutrons using a similar loading method to our work (but different detection scheme) was unsuccessful because of the high temperature of the helium 
during the loading phase 13 .

Crucial to the utility of traps are the techniques used to load them. In order to catch a particle in a static conservative trap, its energy must be lowered while it is in the potential well. Atoms and molecules have been cooled and loaded into magnetic traps by scattering with either cryogenic surfacest4.15, cold gases 16 or photons from a laser beam (laser cooling) 1 . Neutrons, however, cannot be loaded by such methods because they cannot be excited optically and interact too weakly with atoms to be effectively cooled by a gas. Direct thermalization with a cold solid or liquid is generally precluded by the high probability for neutron absorption in the vast majority of materials.

Our trapping of neutrons relies on a loading technique that employs the "superthermal process" 8 . A neutron with kinetic energy near $11 \mathrm{~K}$ (where the free neutron and superfluid helium dispersion curves cross) that passes through the helium-filled trapping region can lose nearly all of its energy through the creation of a single phonon. Neutrons that scatter to energies less than the trap depth $(\leqslant 1 \mathrm{mK})$ and in the appropriate spin state are trapped. Upscattering of UCN in liquid helium by single phonon absorption is highly suppressed due to the low density of $11 \mathrm{~K}$ phonons in the superfluid (proportional to $e^{-11 \mathrm{~K} / T}$ ). The rate of higher-order (multiplephonon) upscattering processes is proportional to $T^{7}$ (ref. 19). The helium is maintained below a temperature of $250 \mathrm{mK}$ to minimize upscattering of the trapped neutrons (total calculated upscattering rate per neutron $\left.<10^{-6} \mathrm{~s}^{-1}\right)$.

Snother possible loss mechanism for trapped neutrons is nuclear absorption due to ${ }^{3} \mathrm{He}$ contamination of the superfluid ${ }^{4} \mathrm{He}$ bath. Unlike ${ }^{4} \mathrm{He}$ which does not absorb thermal neutrons at all, ${ }^{3} \mathrm{He}$ readily absorbs neutrons with a thermal cross section, $\sigma=5330 \times 10^{-24} \mathrm{~cm}^{2}$. To minimize these losses, our superfluid-helium bath was isotopically purified $\left({ }^{3} \mathrm{He} /{ }^{4} \mathrm{He}<5 \times 10^{-13}\right.$; ref. 20), giving a calculated absorption loss rate per neutron of $5 \times 10^{-6} \mathrm{~s}^{-1}$. Thus, the trapped neutrons travel unimpeded through the helium and reside in the trap for a time limited primarily by the beta-decay lifetime of the neutron.

In our experiment, the superfluid helium is contained in a tube that is axially centered within a superconducting magnetic trap (see Fig. 1). The incident cold neutron beam is colli- 
mated by a neutron-absorbing ring, passes through the trapping region, and is absorbed by the beam stop. As the beam traverses the trapping region, about $1 \%$ of the $11 \mathrm{~K}$ neutrons scatter in the helium. Some of these neutrons are trapped and the remainder are absorbed by shielding materials that surround the helium.

When a trapped neutron decays (into an electron, proton and anti-neutrino), the resulting high-energy electron (up to $782 \mathrm{keV}$ ) travels through the helium leaving a trail of ionized helium atoms. These ions quickly combine with neutral helium atoms to form excimer diatomic molecules, about half in singlet states and half in triplet states. About 15 singlet molecules are created per kiloelectronvolt of electron energy and the trail is 0-20 $\mathrm{mm}$ longen. The singlet molecules decay within $10 \mathrm{~ns}$, emitting a pulse of light in the extreme ultraviolet (EUV), $\lambda \approx 70-90 \mathrm{~nm}$. This pulse of scintillation light is the signal of a neutron decaying in the trap. Radiative decay of the triplet states has a much longer lifetime, which we have measured to be $13 \pm 2 \mathrm{~s}$ (ref. 22), and does not contribute to our signal.

The EUV scintillation light is wavelength-shifted to the visible and transported to the outside of the cryostat where it is detected. As shown in Fig. 1, the superfluid-helium-filled trapping region is surrounded by an acrylic tube. The inside surface of this tube is coated with a thin layer of polystyrene doped with the organic fluor tetraphenyl butadiene (TPB; ref. 23). The TPB converts the EUV scintillation light into blue light, a portion of which is internally reflected down the length of the tube 4 . The tube is optically coupled to a solid light guide which transports the light to the end of the $250 \mathrm{mK}$ region. The light then passes through a window at $4 \mathrm{~K}$ and into a second light guide which exits the dewar. The light is split into two guides that are each coupled to a photomultiplier tube (PMT). Background from uncorrelated photons is suppressed by requiring coincident detection of at least two photoelectrons in each PMT. This is required because of significant time-dependent luminescence (initial counting rate without coincidence detection of $\sim 10^{4} \mathrm{~s}^{-1}$ ), induced by neutron absorption in the shielding materials. In a series of calibration runs (using a ${ }^{113} \mathrm{Sn}, 360-\mathrm{keV}$ beta line source placed in the center of the trapping region) we have determined that a total of $14.5 \pm 1.5$ photoelectrons per megaelectronvolt of beta energy are detected in a pulse $20 \mathrm{~ns}$ wide. We estimate that a 
coincidence signal is recorded for $31 \pm 4 \%$ of neutron decays in the trapping region.

In each experimental run, the cold neutron beam is allowed to pass through the trapping region for $1,350 \mathrm{~s}$, after which the beam is blocked and pulses of light are counted for 3,600 s. While the beam is on, neutrons interact with the helium and the number of UCN in the trap increases. After the beam is turned off, the PMTs are turned on and a decreasing rate of scintillation events is observed. A background signal, consisting of both constant and timevarying components, obscures the trapped neutron signal. These backgrounds are subtracted by taking data in two different kinds of run, trapping (signal + background) and non-trapping (background only), and subtracting the two. In a trapping run the magnetic field is on during the initial loading phase, so that UCN are confined in the trap, while in a non-trapping run the magnetic field is initially off, so that no neutrons can be trapped. In a non-trapping run, the field is turned on during detection so that any residual luminescence (which depends on the magnetic field) will be properly subtracted. Equal numbers of each kind of run were taken, pooled and subtracted to give the background subtracted data.

Two sets of background subtracted data were collected: set 1 with a trap depth of $0.76 \mathrm{mK}$ (figure 2a) and set 2 with a lower trap depth of $0.50 \mathrm{mK}$ (figure 2 $\mathrm{c}$ ). (The lower trap depth was used due to problems with the magnet.) Most of the run-to-run variation in background rate is eliminated by excluding the first two pairs of runs in which the background rate is changing quickly due to activation of materials with lifetimes $>12 \mathrm{~h}$. The remaining 23 pairs in set 1 (from about five days of running) and 120 pairs in set 2 (from about three weeks of running) are pooled and modeled as:

$$
W_{1}=\alpha_{1} e^{-t / \tau}+C_{1}, \quad W_{2}=\alpha_{2} e^{-t / \tau}+C_{2}
$$

The subscripts 1 and 2 refer to sets 1 and $2, \alpha_{i}=\epsilon N_{i} / \tau, N_{i}$ is the initial number of trapped neutrons and $i=1$ or $2, \epsilon$ is the detection efficiency, and $\tau$ is the lifetime of neutrons in the trap. The constant $C_{i}$ is present to account for the possible remaining effect of the changing background rate due to long-lived activation. However, in all of our fits the value of $C_{i}$ is consistent with zero. The fit is performed simultaneously on the two data sets, minimizing the 
total $\chi^{2}$ while varying five parameters: $\alpha_{1}, \alpha_{2}, C_{1}, C_{2}$ and $\tau$. The only parameter connecting the two data sets is $\tau$. The best fit values indicate $N_{1}=560 \pm 160$ and $N_{2}=240 \pm 65$. Calculations using the known beam flux, trap geometry and the theory of the superthermal process predict $N_{1}=480 \pm 100$ and $N_{2}=255 \pm 50$, in good agreement with the measured values. The best fit value for the lifetime, $\tau=750_{-200}^{+330} \mathrm{~s}$ is consistent with the presently accepted value of the neutron beta-decay lifetime of $886.7 \pm 1.9 \mathrm{~s}$ (ref. 5). All of the errors quoted correspond to a $68 \%$ confidence interval.

To verify that our signal is due to trapped neutrons, we doped the isotopically pure ${ }^{4} \mathrm{He}$ with ${ }^{3} \mathrm{He}$ at a concentration of $2 \times 10^{-7}{ }^{3} \mathrm{He} /{ }^{4} \mathrm{He}$. This amount of ${ }^{3} \mathrm{He}$ absorbs the trapped neutrons in less than $1 \mathrm{~s}$ without affecting anything else in the experiment (less than $1 \%$ of the $11 \mathrm{~K}$ neutrons are absorbed by ${ }^{3} \mathrm{He}$ ). The ${ }^{3} \mathrm{He}$ background subtracted data (see Fig. 2fb and d) is consistent with zero, and differs from the signal data by more than four standard deviations. This confirms that our signal is due to trapped neutrons.

This work demonstrates the loading, trapping, and detection techniques necessary for performing a neutron lifetime measurement using magnetically trapped UCN. Another important result is the direct confirmation of the theoretical prediction of the UCN density in the trap. Our value for the number of trapped neutrons at a $0.76 \mathrm{mK}$ trap depth corresponds to a density of $2 \mathrm{UCN} / \mathrm{cm}^{3}$, compared to the density of $1 \mathrm{UCN} / \mathrm{cm}^{3}$ obtained in the UCN material bottle experiments 6 目 with a comparable UCN cut-off energy and higher flux reactor. Our measured density is consistant with previous measurements of the UCN production rate based on observation of upscattered UCN at higher temperatures 23 and confirms that the low number of UCN detected in ref. 26 was not due to an intrinsically low UCN production rate.

Magnetic trapping should allow significant improvement in the measurement of the neutron lifetime $\tau_{n}$. There are several loss mechanisms specific to this method, including thermal upscattering and ${ }^{3} \mathrm{He}$ absorption. Both of these can be suppressed to a high level as discussed above. Another possible loss mechanism is neutron depolarization. When the neutron passes through a region where the field changes direction quickly compared to the neutron's spin precession frequency, the neutron can flip spin and be ejected from the trap. In our trap geometry, 
the trap has no zero-field regions and this loss rate can be minimized by the application of a bias field 3 . 27 .

Using our method with a higher-flux source of cold neutrons, a $10^{-5}$ measurement of $\tau_{n}$ should be possiblel. In the near term, improvements such as increased trap size and depth (resluting in an improved ratio of signal-to-background events) should allow an improved accuracy measurement of $\tau_{n}$ at the $5 \times 10^{-4}$ level. Other potential applications of superthermal UCN sources include searches for time-reversal violationes and new methods of neutron scattering especially suited for the study of large (biological-scale) molecules 29 .

Received 5 August; accepted 16 November 1999.

1. Dubbers, D. Test of the standard model with free neutron beta decay. Nucl. Phys. A 527, 239-250 (1991).

2. Lopez, R. E. \& Turner, M. S. Precision prediction for the big-bang abundance of primordial ${ }^{4}$ He. Phys. Rev. D 59, 103502/1-14 (1999).

3. Doyle, J. M. \& Lamoreaux, S. K. On measuring the neutron beta-decay lifetime using ultracold neutrons produced and stored in a superfluid- ${ }^{4}$ He-filled magnetic trap. Europhys. Lett. 26, 253-258 (1994).

4. Phillips, W. D. in Laser Manipulation of Atoms and Ions (eds. Arimondo, E., Phillips, W. \& Sturmia, F.) 289-344 (North Holland, Amsterdam, 1992).

5. Caso, C. et al. Review of Particle Physics. Eur. Phys. J. C 3, 1-794 (1998).

6. Byrne, J. et al. A revised value for the neutron lifetime measured using a Penning trap. Europhys. Lett. 33, 187-192 (1996).

7. Mampe, W., Ageron, P., Bates, C., Pendlebury, J. M. \& Steyerl, A. Neutron lifetime measured with stored ultracold neutrons. Phys. Rev. Lett. 63, 593-596 (1989).

8. Nesvizhevskii, V.V. et al. Measurement of the neutron lifetime in a gravitational trap and analysis of experimental errors. Sov. Phys. JETP 75, 405-412 (1992).

9. Paul, W., Anton, F., Paul, L., Paul, S. \& Mampe, W. Measurement of the neutron lifetime in a magnetic storage ring. Z. Phys. C 45, 25-30 (1989).

10. Golub, R., Richardson, D. \& Lamoreaux, S. K. Ultra-Cold Neutrons (Adam Hilger, Bristol, UK 1991).

11. Vladimirskii, V. V. Magnetic mirrors, channels and bottles for cold neutrons. Sov. Phys. JETP 12, 740-746 (1961). 
12. Abov, Yu. G., Borovlëv, S. P., Vasil'ev, V. V., Vladimirskii, V. V. \& Mospan, E. N. Measurement of the time of storage of ultracold neutrons in a magnetic trap. Sov. J. Nucl. Phys. 38, 70-73 (1983).

13. Niehues, N. Untersuchungen an einer magnetischen Flasche zur Speicherung von Neutronen. (Investigations on a magnetic bottle for the storage of neutrons.) Thesis, Friedrich Wilhelm Univ. Bonn (1983).

14. Hess, H. F. et al. Magnetic trapping of spin-polarized atomic hydrogen. Phys. Rev. Lett. 59, 672-675 (1987).

15. van Roijen, R., Berkhout, J. J., Jaakkola, S. \& Walraven, J. T. M. Experiments with atomic hydrogen in a magnetic trapping field. Phys. Rev. Lett. 61, 931-934 (1988).

16. Doyle, J. M., Friedrich, B., Kim, J. \& Patterson, D. Buffer-gas loading of atoms and molecules into a magnetic trap. Phys. Rev. A 52, R2515-R2518 (1995).

17. Chu, S. in Laser Manipulation of Atoms and Ions (eds. Arimondo, E., Phillips, W. \& Sturmia, F.) 239-288 (North Holland, Amsterdam, 1992).

18. Golub, R. \& Pendlebury, J. M. The interaction of ultra-cold neutrons (UCN) with liquid helium and a superthermal UCN source. Phys. Lett. A 62, 337-339 (1977).

19. Golub, R. et al. Operation of a superthermal ultracold neutron source and the storage of ultracold neutrons in superfluid helium-4. Z. Phys. B 51, 187-193 (1983).

20. Hendry, P. C. \& McClintock, P. V.E. Continuous flow apparatus for preparing isotopically pure ${ }^{4}$ He. Cryogenics 27, 131-138 (1987).

21. Adams, J. S., Kim, Y.H., Lanou, R.E., Maris, H. J. \& Seidel, G. M. Scintillation and quantum evaporation generated by single monoenergetic electrons stopped in superfluid helium. J. Low Temp. Phys. 13, 1121-1128 (1998).

22. McKinsey, D. N. et al. Radiative decay of the metastable $\mathrm{He}_{2}\left(\mathrm{a}^{3} \Sigma_{\mathrm{u}}^{+}\right)$molecule in liquid helium. Phys. Rev. A 59, 200-204 (1999).

23. McKinsey, D. N. et al. Fluorescence efficiencies of thin scintillating films in the extreme ultraviolet spectral region. Nucl. Inst. and Meth. B 132, 351-358 (1997).

24. Habicht, K. Szintillationen in flüssigem Helium - ein Detektor für ultrakalte Neutronen. (Scintillations in liquid helium - a detector for ultracold neutrons.) Thesis, Tech. Univ. Berlin (1998).

25. Kilvington, A. I., Golub, R., Mampe, W. \& Ageron, P. Scattering of ultra-cold neutrons (UCN) by superfluid helium at temperatures around 1 K. Phys. Lett. A 125, 416-420 (1987).

26. Yoshiki, H. et al. Observation of ultracold-neutron production by 9 - $\AA$ cold neutrons in superfluid helium. Phys. Rev. Lett. 68 1323-1326 (1992).

27. Happer, W. Optical Pumping. Rev. Mod. Phys. 44, 169-249 (1972). 
28. Golub, R. \& Lamoreaux, S. K. Neutron electric-dipole moment, ultracold neutrons and polarized ${ }^{3}$ He. Phys. Rep. 237, 1-62 (1994).

29. Golub, R. Ultracold neutrons: their role in studies of condensed matter. Rev. Mod. Phys. 68, 329-347 (1996).

30. Butterworth, J. S. et al. A removable cryogenic window for transmission of light and neutrons. Rev. Sci. Inst. 69, 3998-3999 (1998).

Acknowledgments. We thank J. M. Rowe, D. M. Gilliam, G. L. Jones, J. S. Nico, N. Clarkson, G. P. Lamaze, C. Chin, C. Davis, D. Barkin, A. Black, V. Dinu, J. Higbie, H. Park, R. Ramakrishnan, I. Siddiqi, and G. Brandenburg for their help with this project. We thank P. McClintock, D. Meredith and P. Hendry for supplying the isotopically pure helium. We acknowledge the support of the NIST, US DOC, in providing the neutron facilities used in this work. This work is supported in part by the US NSF. The NIST authors acknowledge the support of the US DOE.

Correspondence and requests for materials should be addressed to P.R.H. (e-mail: paul.huffman@nist.gov). 


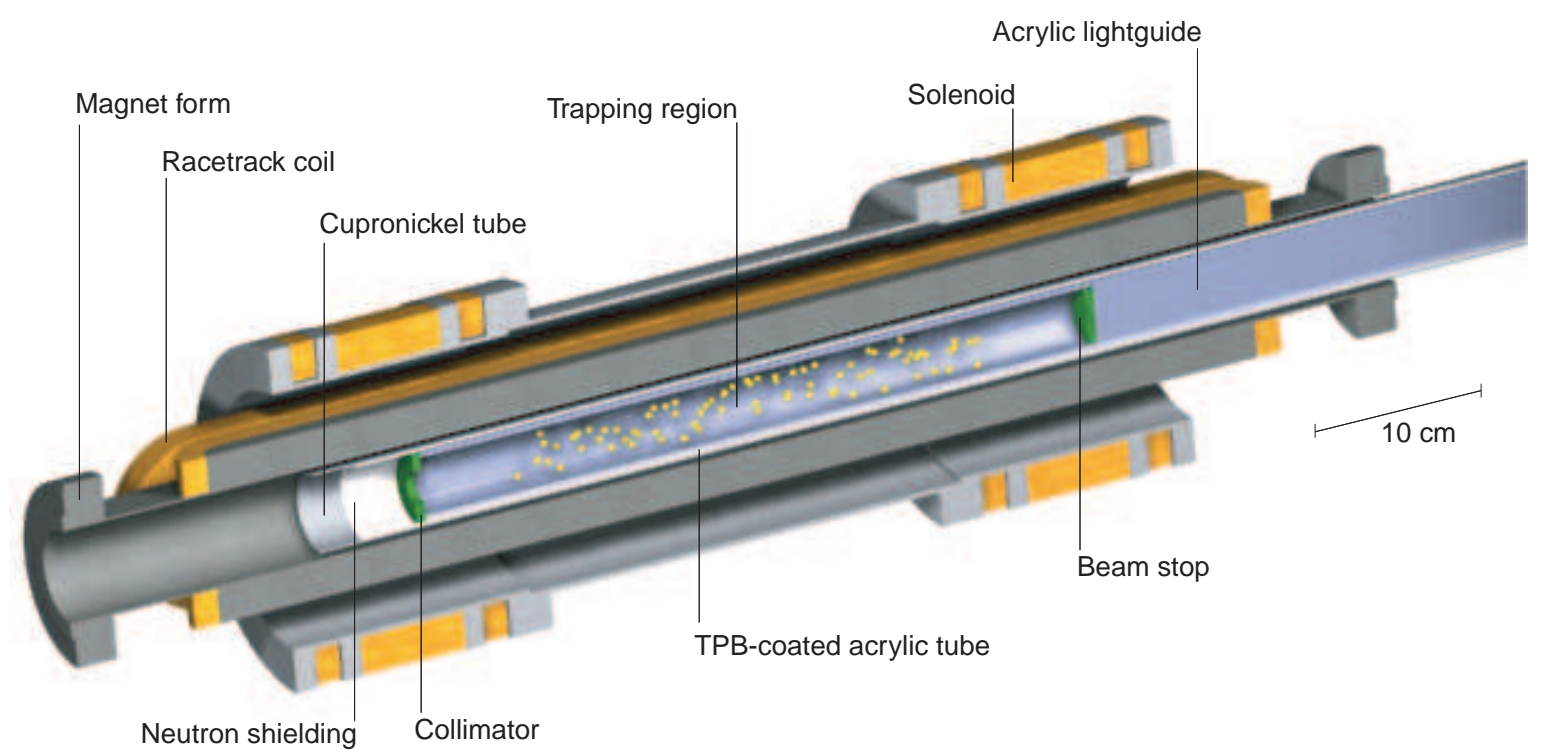

Figure 1: Half section view of the neutron trapping apparatus. The trapping region is filled with isotopically pure ${ }^{4} \mathrm{He}$ at a temperature $\leqslant 250 \mathrm{mK}$. The helium is contained within a cupronickel tube. A beam of cold neutrons passes through a series of teflon windows 30 and enters the helium from the left. It is collimated by a boron carbide ring, passes through the trapping region and is absorbed by a boron carbide beam stop. Approximately $1 \%$ of the $11 \mathrm{~K}$ neutrons scatter in the superfluid helium. Those neutrons (yellow) in the low-field-seeking spin state and with energy below the trap depth are magnetically confined. The rest of the scattered neutrons are absorbed by neutron shielding material (boron nitride) surrounding the trapping region. The magnetic trapping field is created by an assembly of superconducting magnets. Radial confinement is provided by a quadrupole constructed from four racetrack-shaped coils and axial confinement is provided by two sets of solenoids. Electrons from neutron beta-decay cause EUV scintillations in the superfluid helium which are wavelength shifted to the visible by a thin film of TPB-doped 3 polystyrene coated on the inside of an acrylic tube surrounding the trapping region. This tube is optically coupled to an acrylic lightguide which transports the blue light to the end of the $250 \mathrm{mK}$ region (to the right). 


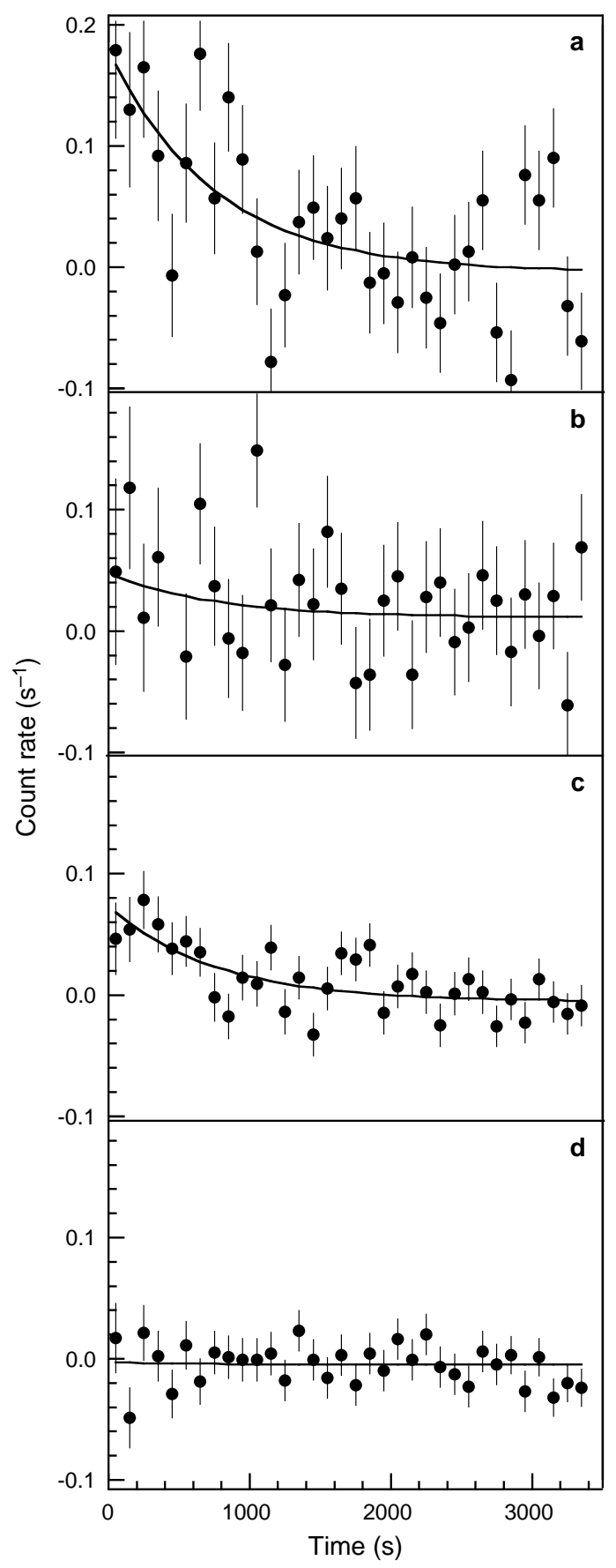

Figure 2: Counting rate as a function of time after the neutron beam is turned off (pooled background subtracted data). The two sets of trapping data are fit for five parameters: $\alpha_{1}, \alpha_{2}, C_{1}$, $C_{2}$ and $\tau$. The constant offset terms, $C_{1}$ and $C_{2}$ are found to be $-0.004 \pm 0.015$ and $-0.006 \pm 0.006$ respectively, both consistent with zero. The best fit values for the initial counting rates for trapping data sets 1 and 2 are $\alpha_{1}=0.196 \pm 0.05 \mathrm{~s}^{-1}$ and $\alpha_{2}=0.084 \pm 0.02 \mathrm{~s}^{-1}$. The detection efficiency of $\epsilon=31 \pm 4 \%$ combined with the known neutron lifetime, gives $N_{1}=560 \pm 160$ and $N_{2}=240 \pm 65$. The best fit value for the lifetime is $\tau=750_{-200}^{+330} \mathrm{~s}$. The results of this fit are shown on plots $\mathbf{a}$ and $\mathbf{c}$. The ${ }^{3} \mathrm{He}$ data is fit to four parameters, $\alpha_{1}, \alpha_{2}, C_{1}$, $C_{2}$, with $\tau$ fixed at $750 \mathrm{~s}$. Because no fit parameter applies to both data sets, this is equivalent to two separate two-parameter fits, one to each data set. The amplitudes, $\alpha_{1}^{\mathrm{He}}=0.040 \pm 0.045$ and $\alpha_{2}^{\mathrm{He}}=$ $0.005 \pm 0.016$, are both consistent with zero. The constant offset terms are $C_{1}=0.011 \pm 0.011$ and $C_{2}=-0.006 \pm 0.004$. The ${ }^{3} \mathrm{He}$ data and fit are shown on plots $\mathbf{b}$ and $\mathbf{d}$. (a) Trapping data set 1 (23 pairs of runs at a trap depth of $0.76 \mathrm{mK}$ ). (b) ${ }^{3} \mathrm{He}$ data set 1 (20 pairs at $0.76 \mathrm{mK}$ ). (c) Trapping data set 2 (120 pairs at $0.50 \mathrm{mK})$. (d) ${ }^{3} \mathrm{He}$ data set 2 (125 pairs at $0.50 \mathrm{mK}$ ). 